\title{
Reviewers 1997
}

\author{
Accand, Janet \\ Avery, Chris \\ Bakker, Connie \\ Berger, Barbara \\ Blixrud, Julia \\ Bloss, Alex \\ Bogan, Ruth \\ Boissonnas, Christian \\ Boomgaarden, Wes \\ Bradsher, Carol \\ Budd, John \\ Calhoun, Karen \\ Childress, Eric \\ Chou, Yu-lan \\ Christian \\ Chrzastowski, Tina \\ DeStefano, Paula \\ Diedrichs, Carol \\ Dirks, Lee \\ Dole, Wanda \\ Drewes, Jeanne \\ Duke, John \\ Geer-Butler, Beverley \\ Gertz, Janet \\ Gould, Linda \\ Haberman, Marge \\ Harrison, S.E. \\ Hazen, Dan \\ Hearn, Stephen \\ Hemmasi, Harriette \\ Hixon, Carol \\ Holley, Bob \\ Izbicki, Thomas \\ Jacob, Elin \\ Jedlicka, Beth \\ Johns, Cecily
}

Johnston, George

Jordan, Sonja

Kaplan, Michael

Kellerman, Lydia

Kohberger, Paul

Lambrecht, Jay

Lucas, Diane

MacEwan, Bonnie

Martin, Sylvia

McCombs, Gillian

McDonough, Joyce

McEwan, Bonnie

McGarry, Dorothy

Mischo, Bill

Motylewski, Karen

Reed, Helen

Rice, Janice Beaudin

Rider, Mary

Riemer, John

Ruschoff, Carlen

Schmidt, Karen

Sinclair, Regina

Strawn, Gary

Su, Meng-fen

Sun, Dajin

Tsao, Jai-hsya

Van Deventer, Barbara

Vizine-Goetz, Diane

Weintraub, Tamara

Wilder, Stanley

Williamson, Nancy

Winters, Barbara

Wolven, Bob

Woods, Larry

Wool, Gregory

Zabel, Diane 